OPEN

SUBJECT AREAS:

BIOLOGICAL PHYSICS

COMPUTATIONAL BIOPHYSICS

PHOTONIC DEVICES

APPLIED PHYSICS

Received

4 April 2013

Accepted

24 June 2013

Published

15 July 2013

Correspondence and requests for materials should be addressed to

N.J. (njohnson@ physics.miami.edu) or F.C. (felipe.caycedo@ uni-ulm.de)

\section{Extreme alien light allows survival of terrestrial bacteria}

\author{
Neil Johnson' ', Guannan Zhao', Felipe Caycedo ${ }^{2}$, Pedro Manrique' ', Hong Qi' ', Ferney Rodriguez ${ }^{3}$ \\ \& Luis Quiroga ${ }^{3}$
}

'Physics Department, University of Miami, Florida FL 33126, U.S.A., '2Department of Physics, Universitat Ulm, Germany,
${ }^{3}$ Departamento de Fisica, Universidad de Los Andes, Bogota, Colombia.

Photosynthetic organisms provide a crucial coupling between the Sun's energy and metabolic processes supporting life on Earth. Searches for extraterrestrial life focus on seeking planets with similar incident light intensities and environments. However the impact of abnormal photon arrival times has not been considered. Here we present the counterintuitive result that broad classes of extreme alien light could support terrestrial bacterial life whereas sources more similar to our Sun might not. Our detailed microscopic model uses state-of-the-art empirical inputs including Atomic Force Microscopy (AFM) images. It predicts a highly nonlinear survivability for the basic lifeform Rsp. Photometricum whereby toxic photon feeds get converted into a benign metabolic energy supply by an interplay between the membrane's spatial structure and temporal excitation processes. More generally, our work suggests a new handle for manipulating terrestrial photosynthesis using currently-available extreme value statistics photon sources.

hotosynthetic organisms ${ }^{1-9}$ are among the oldest lifeforms, and as such feature prominently in current debates about the possibility of life elsewhere in the universe ${ }^{10,11}$, alien life on Earth ${ }^{12}$ - and more generally, as potential renewable energy sources ${ }^{13}$. Among the recent advances in photosynthesis research ${ }^{1,2,13-20}$ are state-of-the-art AFM maps of the light-harvesting (LH) complexes in bacterial membranes ${ }^{1}$, and improved estimates for the relevant excitation timescales ${ }^{21,22}$. Both are essential ingredients for any realistic model of lightharvesting dynamics. Our model for excitation migration in purple bacterium Rsp. Photometricum, an archetypal terrestrial photosynthetic organism, inputs directly large spatial AFM network maps containing approximately 400 complexes (see Fig. 1 and Supplementary Information (SI)) together with these improved excitation timescales. It is well-known that certain environmental extremes would hinder the survival of a photosynthetic bacterium, and hence that certain exoplanets would be less likely to support terrestrial photosynthetic life than others - however we know of no prior studies calculating the effect of incident photon arrival times. This is in part because of the previous lack of detailed information concerning the LH network and excitation timescales, but also because the prevailing view holds that it suffices to find planets with a similar incident light intensity.

The time-series of the incident photons creating excitations in the membrane, $\gamma(t)$, and the quinol output from the RC, $\phi(t)$, can both be characterized statistically by their burstiness $B$ and memory $M$, following Goh and Barabasi ${ }^{23}$. Random arrival with constant probability per unit time constitutes a Poisson process and yields an exponential distribution $P(\tau)$ for the time interval $\tau$. Deviations from this exponential hence provide a measure of the burstiness: $B \equiv\left(\sigma_{\tau} / m_{\tau}-1\right) /\left(\sigma_{\tau} / m_{\tau}+1\right)$ where $m_{\tau}$ and $\sigma_{\tau}$ are the mean and standard deviation respectively of the distribution $P(\tau)$. The memory $M$ between consecutive intervals is given by ${ }^{23}: M \equiv \frac{1}{n_{\tau}-1}$ $\sum_{i=1}^{n_{\tau}-1}\left(\tau_{i}-m_{1}\right)\left(\tau_{i+1}-m_{2}\right) / \sigma_{1} \sigma_{2}$ where $n_{\tau}$ is the number of time intervals and $m_{1}\left(m_{2}\right)$ and $\sigma_{1}\left(\sigma_{2}\right)$ are the sample mean and standard deviation of the $\tau_{i}\left(\tau_{i+1}\right)$ 's respectively $\left(i=1, \ldots, n_{\tau}-1\right)$. By definition, $-1 \leq M \leq 1$ and -1 $\leq B \leq 1$. The $\gamma(t)$ for thermal sources such as our own Sun is a Poisson-like process with $(M, B) \approx(0,0)$, and produces an RC output $\phi(t)$ which also has $(M, B) \approx(0,0)$. However non-thermal sources, such as that recently produced in laboratory silicon systems using stimulated and coherent anti-Stokes Raman scattering ${ }^{24}$, would not be restricted to having $(M, B) \approx(0,0)$ and hence merit investigation. For simplicity our model considers each arriving photon as creating one initial excitation: This avoids adding another layer of statistical analysis for the absorption and does not change our main conclusions.

\section{Results}

Figures 2 and 3 present our model's predictions for the survivability of Rsp. Photometricum using three simple inputs whose $(M, B)$ values can be calculated exactly analytically, thereby avoiding questions of finite sample size 


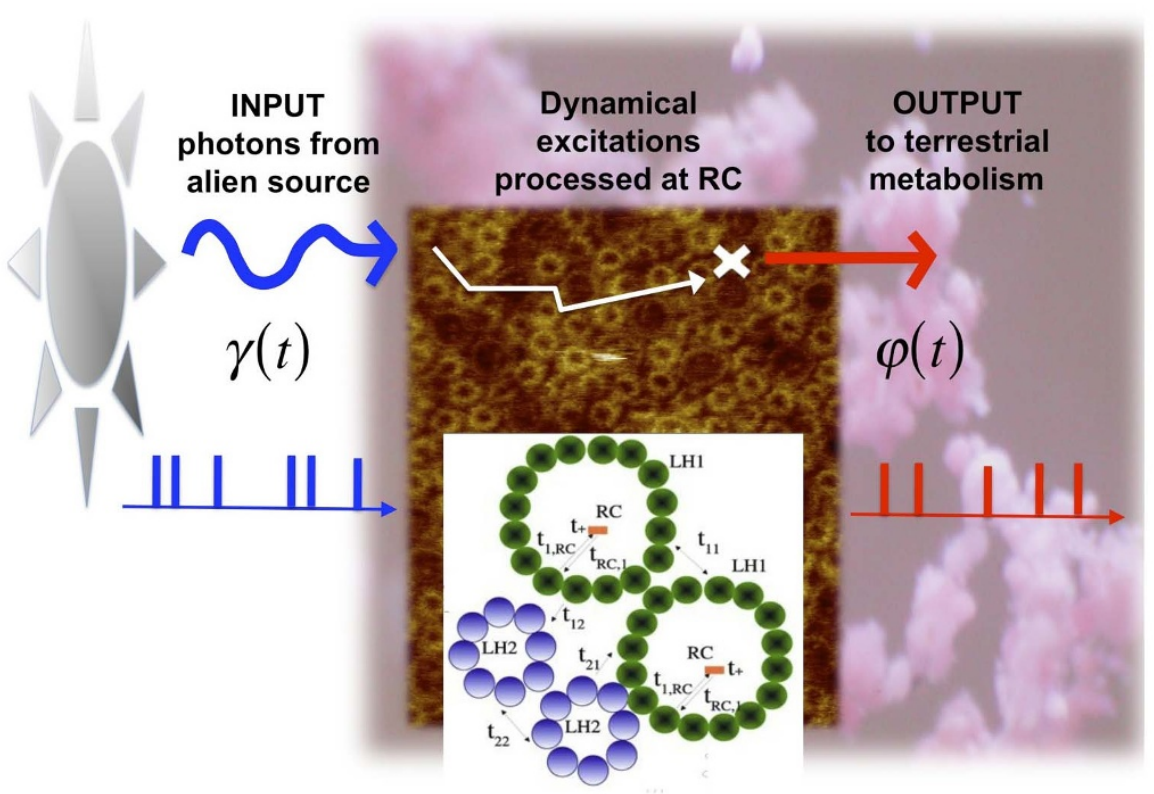

Figure $1 \mid$ Our model. Incident photon train produces excitations in purple bacteria $(\gamma(t))$ which then migrate across a large network containing approximately 400 light-harvesting complexes. Excitations are then processed at the reaction centers (RC) producing quinol output $(\phi(t))$ for chemical metabolism. The AFM structure of the light-harvesting network adapted from Ref. 1, is used as an input to our model. Background shows purple bacteria colony (images courtesy of Drs. W. Lanier and J. Sturgis). Relevant timescales for exciton and charge separation dynamics are shown.

(a)

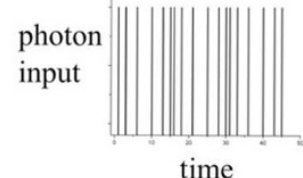

(b)

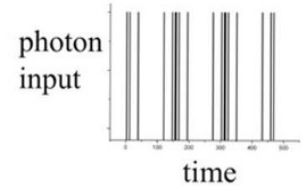

(c)

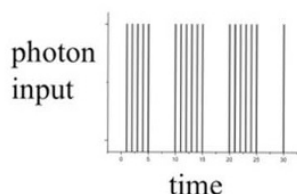

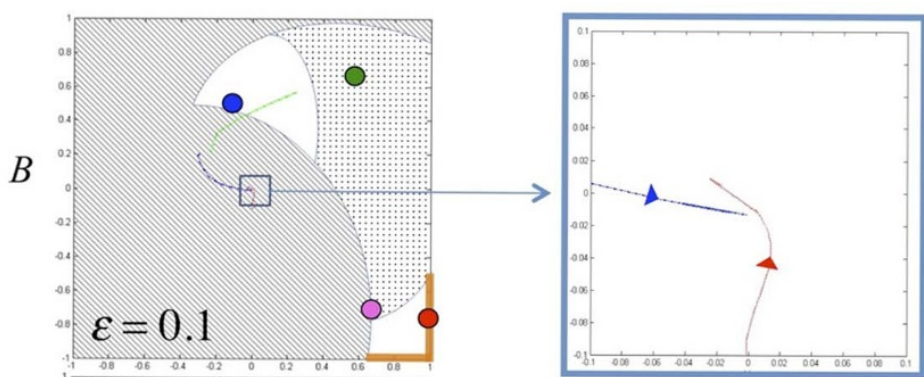
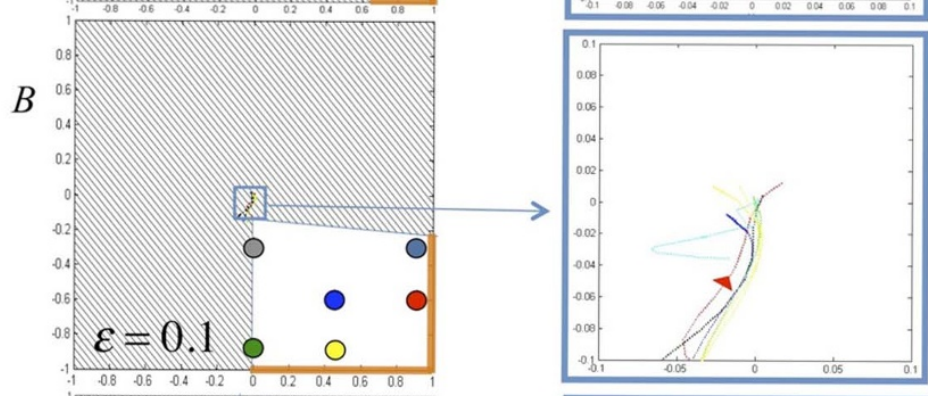

$B$
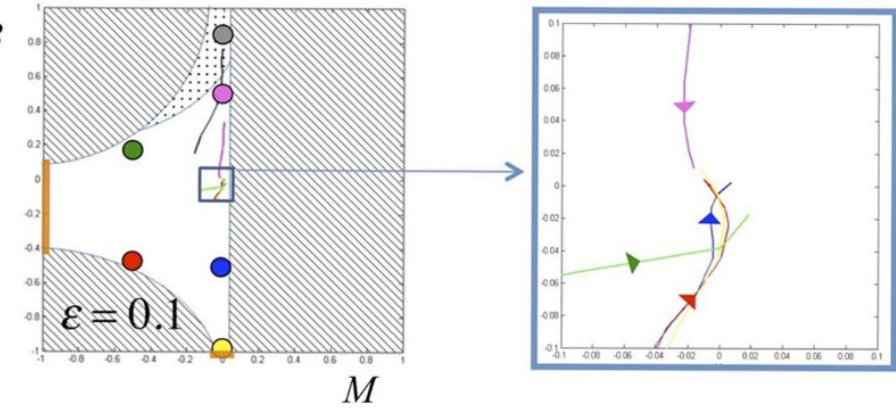

Figure $2 \mid$ Survivability of terrestrial bacteria. Three simple input time-series $\gamma(t)$ are considered as illustration, with the sum of the white and dotted areas representing possible burstiness $B$ and memory $M$ values for that input process. Since the output $\phi(t)$ needs to be similar to on Earth (i.e.

$(|M|,|B|) \leq(\epsilon, \epsilon)$ where $\epsilon=0.1$ ), white regions correspond to $\gamma(t)$ inputs for which the bacteria would in principle survive while dotted regions correspond to $\gamma(t)$ inputs for which survival is less favorable. Trajectories show the $(M, B)$ output values obtained as the range of physically reasonable RC closure times is spanned, for a particular $(M, B)$ input indicated by a circle of the same color. Right-hand column shows magnified version. Photon inputs correspond to (a) bunched input, (b) power-law step input and (c) step input. 
(a)

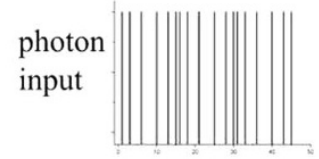

time

(b)

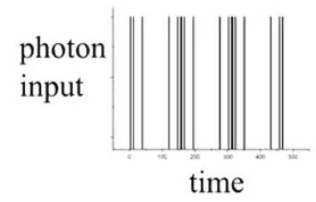

(c)

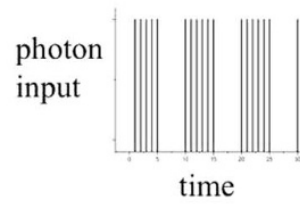

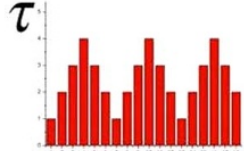

photon number

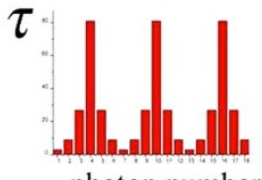

photon number

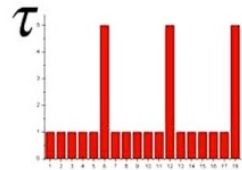

photon number

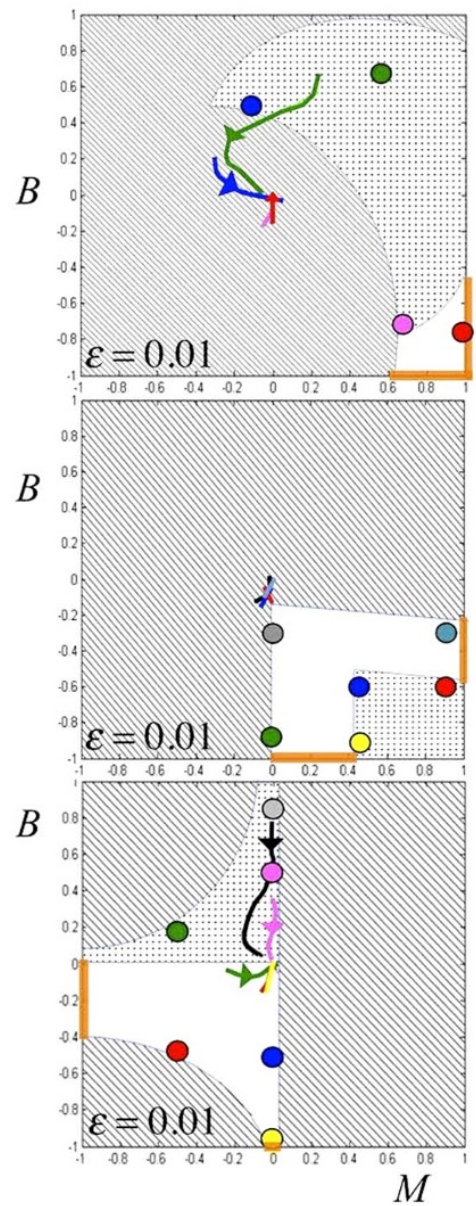

Figure 3 As in Fig. 2, but now the condition for survival is more stringent. $\epsilon=0.01$. Additional histograms show waiting times $\tau$ between the incident photon arrivals.

and hence boosting the robustness of the results. Although for each there are some portions of the $(M, B)$ space that cannot be generated (indicated by diagonal shading), taken collectively they cover almost the entire $(M, B)$ space. Each trajectory of a given color represents the possible RC outputs $\phi(t)$ generated using a particular input $(M, B)$ value (denoted by a circle of the same color), but with RC closing times chosen from anywhere in the empirically valid range (i.e. 20 to $1000 \mathrm{~Hz}$ ). If for one of these RC closing times, part of this trajectory lies within some small range $\epsilon$ of $(0,0)$ in the $(M, B)$ plot and hence provides a $\phi(t)$ similar to that on Earth, a terrestrial bacteria with that value of the RC closing time would experience a quinol output from the RC which is identical to that on Earth. All other conditions being equal, it would therefore be likely to survive since its metabolism experiences the same quinol supply chain as on Earth. The corresponding colored circle therefore appears in the white region of the $(M, B)$ space denoting survivability. If the trajectory does not lie close to $(0,0)$ in the $(M, B)$ plot, then the quinol supply to the metabolism is bursty, which means that quinol molecules would sporadically bunch around the RC. It is known that such molecular crowding can alter the course of biochemical reactions ${ }^{25}$, which in turn suggests the bacterium's survival would be less likely. The corresponding colored circle now lies in a dotted region. We assume that the terrestrial bacteria's exposure to $\gamma(t)$ is too abrupt for its membrane structure to adapt, leaving the possibility of mutations and evolutionary effects for future study. The irregular form of these white survivability regions is due to the highly nonlinear nature of the kinetic interplay between exciton migration and RC trapping, meaning extremes with $|B|$ or $|M|$ equal to 1 may allow survival (e.g. orange lines) while more Earth-like regions (i.e. $M$ and $B$ closer to zero) may not. Figure 3 considers a more stringent condition than Fig. 2 for the output range necessary for terrestrial bacteria survival, hence the smaller white regions.

\section{Discussion}

Further specification of the bacterium's likely survival will require more detailed empirical information about the environmental and biochemical changes that a non-thermal light source such as in Ref. 24 would induce. Our results simply provide a proof-of-principle that, all other conditions being equal, light sources with extreme photon statistics ${ }^{24}$ could allow survival of the photosynthetic bacterium (white regions). In particular, since the temperature of objects receiving energy from such a source depends only on the timeaverage incident energy flux, one can vary the photon arrival statistics without changing the light intensity and hence without changing the temperature of the bacterium or its environment. If an extraterrestrial environment were identified with an Earth-like incident energy flux, temperature, humidity etc., the photon statistics could therefore make the difference between life and death. Our results are also relevant to the scenario of our own sun suddenly developing extremal temporal statistics, e.g. through sudden instabilities in the plasma flow dynamics modulating the irradiance properties at the Earth's surface ${ }^{26}$; the scenario of using artificial light sources as in Ref. 24 to manipulate photosynthesis; and the scenario where the bacteria's chromatophores are artificially modified in such a way that the absorption of our sun's photons takes on statistical properties with $M$ $\neq 0$ and $B \neq 0$. Our results may also help with engineering designerharvesting structures having charge transfer times possessing a particular desired statistic. 


\section{Methods}

We model the excitation migration process as shown in Fig. 1, with details in the Supplementary Information (SI). The transfer rate values that we use (Fig. 1) are measured from pump-probe experiments and agree with generalized Förster calculated rates $^{18}$, assuming intra-complex delocalization. The LH2 $\rightarrow$ LH1 transfer is estimated as $t_{21}=3.3 \mathrm{ps}^{27}$, while LH2 $\rightarrow \mathrm{LH} 2$ transfer timescale is $t_{22}=10 \mathrm{ps}^{18}$. The $\mathrm{LH} 1 \rightarrow \mathrm{LH} 1$ mean transfer time $t_{11}$ has been calculated using a generalized Förster interaction $^{6}$ as $20 \mathrm{ps}$, while the back-transfer LH1 $\rightarrow$ LH2 is approximately $t_{12}=$ $15.5 \mathrm{ps}$. The lowest exciton state is thought to cause the $\mathrm{LH} 1 \rightarrow \mathrm{RC}$ (reaction center) transfer at $77 \mathrm{~K}$, whose non-vanishing dipole strength arises from ring symmetry breaking. Involvement of the bright second and third low lying states at higher temperatures, improves the transfer time to $t_{1 R C}=25 \mathrm{ps}^{22,28}$. Back-transfer from an RC's fully populated lowest exciton state to the second and third bright LH1 states, occurs in a calculated time of $t_{R C 1}=8.1 \mathrm{ps}^{29}$. This is close to the empirically determined value 7-9 $\mathrm{ps}^{30}$. Charge separation at the RC complex will occur within $t_{+}=$ 3 ps depending on whether a charge carrier is available, i.e. the $\mathrm{RC}$ is in an open state. In the RC, two electron transfer steps produce quinol $\left(Q_{B} H_{2}\right)$ after which the $\mathrm{RC}$ is then closed for a few milliseconds before quinol undocks and an available quinone $Q_{B}$ replaces it. This reinitiates the charge carrier cycle and sets the RC in an open state. A proton gradient is generated across the periplasm and cytoplasm, inducing a voltage difference across the chromatophore membrane and hence enabling activation of the bacterial metabolism.

1. Scheuring, S. \& Sturgis, J. N. Chromatic adaptation of photosynthetic membranes. Science 309, 484-487 (2005).

2. Abbott, D., Davies, P. C. \& Pati, A. K. Quantum Aspects of Life (Imperial College Press, 2008).

3. Cogdell, R. J. \& Mullineaux, C. Photosynthetic light harvesting - introduction. Photosynthesis Research 95, 117 (2008).

4. Blankenship, R. Molecular Mechanisms of Photosynthesis (Wiley-Blackwell, 2002).

5. Fleming, G. R. \& van Grondelle, R. Femtosecond spectroscopy of photosynthetic light-harvesting systems. Curr. Opin. Struct. Biol. 7, 738-748 (1997).

6. Ritz, T., Park, S. \& Schulten, K. Kinetics of Excitation Migration and Trapping in the Photosynthetic Unit of Purple Bacteria. Chem Phys Chem 3, 243-248 (2002).

7. Scholes, G., Fleming, G., Olaya-Castro, A. \& van Grondelle, R. Lessons from nature about solar light harvesting. Nature Chem. 3, 763-774 (2011). DOI:10.1038/nchem.1145.

8. Van Amerongen, H., Valkunas, L. \& van Grondelle, R. Photosynthetic Excitons (World Scientific, 2000).

9. Scheuring, S. et al. Watching the photosynthetic apparatus in native membranes. Proc. Natl. Acad. Sci. 101, 11293-11297 (2004)

10. Von Bloh, B. \& Cuntz, F. The Habitability of Super-Earths in Gliese 581. Astronomy and Astrophysics 476, 1365 (2007). Available at http://arxiv.org/pdf/ 0705.3758.pdf. Last accessed on 3-22-2013.

11. Barclay, T. et al. A sub-Mercury-sized exoplanet. Nature 494, 452454 (2013). DOI:10.1038/nature11914.

12. Hoover, R. B. Fossils of Cyanobacteria in CI1 Carbonaceous Meteorites. Journal of Cosmology 13, 1-41 (2011)

13. Niedzwiedzki, D. M. et al. Spectroscopic studies of two spectral variants of lightharvesting complex 2 (LH2) from the photosynthetic purple sulfur bacterium Allochromatium vinosum. Biochimica et Biophysica Acta: BBA Bioenergetics 1817, 1576-1587 (2012). ISSN 0005-2728. DOI:10.1016/j.bbabio.2012.05.009.

14. Chin, A. W. et al. The role of non-equilibrium vibrational structures in electronic coherence and recoherence in pigmentprotein complexes. Nature Physics $\mathbf{9}$, 113118. DOI:10.1038/NPHYS2515.

15. Olaya-Castro, A., Lee, C., Olsen, F. \& Johnson, N. Efficiency of energy transfer in a light-harvesting system under quantum coherence. Phys. Rev. B 78, 085115-7 (2008).

16. Caycedo-Soler, F., Rodriguez, F. J., Quiroga, L. \& Johnson, N. F. Light-Harvesting Mechanism of Bacteria Exploits a Critical Interplay between the Dynamics of Transport and Trapping. Phys. Rev. Lett. 104, 158302-4 (2010); Interplay between excitation kinetics and reaction-center dynamics in purple bacteria. New Journal of Physics 12, 095008-9 (2010). DOI:10.1088/1367-2630/12/9/095008.

17. Fassioli, F., Olaya-Castro, A., Scheuring, S., Sturgis, J. N. \& Johnson, N. F. Energy Transfer in Light-Adapted Photosynthetic Membranes: From Active to Saturated Photosynthesis. Biophysical Journal 97, 2464-2673 (2009).

18. Hu, X., Ritz, T., Damjanović, A., Autenrieth, F. \& Schulten, K. Photosynthetic apparatus of purple bacteria. Q. Rev. of Biophys. 35, 1-62 (2002).

19. Lee, H., Cheng, Y. C. \& Fleming, G. R. Coherence Dynamics in Photosynthesis: Protein Protection of Excitonic Coherence. Science 316, 1462-1465 (2007).

20. Engel, G. S. et al. Evidence for wavelike energy transfer through quantum coherence in photosynthetic systems. Nature 446, 782-786 (2007).

21. Strumpfer, J. \& Schulten, K. Light harvesting complex II B850 excitation dynamics. J. Chem. Phys. 131, 225101-9 (2009). DOI: 10.1063/1.3271348.

22. Strumpfer, J. \& Schulten, K. Excited state dynamics in photosynthetic reaction center and light harvesting complex 1. J. Chem. Phys. 137, 065101-8 (2012).

23. Goh, K. I. \& Barabasi, A. L. Burstiness and memory in complex systems. Europhysics Letters 81, 48002-5 (2008).

24. Borlaug, D., Fathpour, S. \& Jalali, B. Extreme Value Statistics in Silicon Photonics. IEEE Photonics Journal 1, 33-39 (2009).

25. Schnell, S. \& Turner, T. E. Reaction kinetics in intracellular environments with macromolecular crowding: simulations and rate laws. Progress in Biophysics and Molecular Biology 85, 235-260 (2004). doi:10.1016/j.pbiomolbio.2004.01.012.

26. Nandy, D., Muñoz Jaramillo, A. \& Martens, C. H. The unusual minimum of sunspot cycle 23 caused by meridional plasma flow variations. Nature 471, 80-82 (2011).

27. Hess, S. et al. Temporally and spectrally resolved subpicosecond energy transfer within the peripheral antenna complex (LH2) and from LH2 to the core antenna complex in photosynthetic purple bacteria. Proc. Natl. Acad. Sci. USA 92, 12333-12337 (1995).

28. van Grondelle, R., Dekker, J. P., Gillbro, T. \& Sundström, V. Energy transfer and trapping in photosynthesis. Biochim. Biophys. Acta 1187, 1-65 (1994).

29. Damjanović, A., Ritz, T. \& Schulten, K. Excitation energy trapping by the reaction center of Rhodobacter Sphaeroides. Int. J. Quantum Chem. 77, 139-151 (2000).

30. Timpmann, K., Zhang, F. G., Freiberg, A. \& Sundström, V. Detrapping of excitation energy from the reaction center in the photosynthetic purple bacterium Rhodospirillum rubrum. Biochim. Biophys. Acta 1183, 185-193 (1993).

\section{Acknowledgements}

F.J.R. and L.Q. acknowledge financial support from the Facultad de Ciencias and Vicerrectoria de Investigaciones, Universidad de los Andes, through the project "Quantum control of non-equilibrium hybrid systems" (2012-2014). F.C. thanks the Alexander von Humboldt Foundation for funding.

\section{Author contributions}

All authors participated in discussions of the research, its findings, and the content of the manuscript. N.J. and F.C. wrote the manuscript. All authors helped design the research. G.Z. and F.C. did the numerical calculations and G.Z. and N.J. prepared the figures. All authors reviewed the manuscript.

\section{Additional information}

Supplementary information accompanies this paper at http://www.nature.com/ scientificreports

Competing financial interests: The authors declare no competing financial interests.

How to cite this article: Johnson, N. et al. Extreme alien light allows survival of terrestrial bacteria. Sci. Rep. 3, 2198; DOI:10.1038/srep02198 (2013).

This work is licensed under a Creative Commons AttributionNonCommercial-NoDerivs 3.0 Unported license. To view a copy of this license, visit http://creativecommons.org/licenses/by-nc-nd/3.0 